\title{
LA GUYANE: Un département francais comme les autres?'
}

\author{
par Frank Schwarzbeck
}

Après l'indépendance de Bélize (ex Honduras britannique) en 1981, la Guyane Française reste aujourd'hui le seul pays continental en Amérique Latine, Afrique et Asie, ayant une étendue non négligeable (comme le Portugal ou la Hongrie), qui demeure entièrement attachée à un Etat européen. Juridiquement, le territoire français se prolonge en Amérique du Sud et en font partie, au même titre, les autres DOM et TOM ainsi que la Collectivité Territoriale de Mayotte. ${ }^{2}$ Tandis que la possession des îles d'outre-mer est encore un phénomène qui ne se limite pas à la France mais touche aussi les Etats-Unis et la Grande-Bretagne (quoique avec des modalités différentes), le "cas continental" de la Guyane est désormais unique dans le Tiers-Monde. ${ }^{3}$ Cette seule raison paraît suffire pour soulever plusieurs questions.

Outre cette particularité générale, le cas guyanais est intéressant à plus d'un titre. La Guyane fait partie des petits pays du Tiers-Monde dont le nombre s'est accru d'année en année. Il n'est pas surprenant que les sciences sociales et politiques se soient intéressées de plus en plus aux problèmes de ces pays, en posant la question de leur "viabilité". L'indé-

* Cet article est publié conjointement par: Verfassung und Recht in Ubersee et Amérique Latine, No 12, oct.déc. 1982, Paris.

1 Le présent article s'inspire des réflexions développées de façon plus détaillée dans une thèse de doctorat ("Französisch-Guayana. Die letzte kontinentale Uberseebesitzung in Lateinamerika" / "La Guyane Française. La dernière possession d'outre-mer continentale en Amérique Latine“) présentée en 1981 à l'Université de Hambourg et éditée en 1982 par les "Heidelberger Dritte Welt Studien" (Esprint-Verlag, Bergheimerstr. 147, 6900 Heidelberg).

Cette étude n'en constitue pas un résumé mais s'efforce plutôt de mettre l'accent sur quelques problèmes jusqu'à présent peu abordés dans des travaux de langue française.

2 Les départements d'outre-mer et les territoires d'outre-mer se distinguent surtout de deux façons: Tandis que les TOM peuvent manifester (par délibération de leur assemblée territoriale) la volonté de changer de statut et de devenir soit départements d'outre-mer, soit états indépendants (art. 76 de la Constitution de la Ve République), les DOM, eux, n'ont pas cette option. Tout changement de leur statut, à l'exception de leur conversion en des "Collectivités Territoriales" par la loi (art. 72), devrait passer par une révision de la constitution (art. 89) ou par les dispositions de son article 11, autorisant le Président de la République à soumettre au référendum tout projet de loi portant sur l'organisation des pouvoirs publics.

En plus, il y a des différences dans l'organisation institutionelle entre les DOM et les TOM, les derniers jouissant de plus d'autonomie locale. Ayant chacun leur propre statut, les TOM vivent sous "le régime de la spécialité législative": Les lois de la République ne leur sont étendues que sur mention expresse, alors qu'elles s'appliquent immédiatement dans les départements d'outre-mer. Le régime législatif et l'organisation administrative des DOM, en principe calqués sur ceux de la métropole, peuvent toutefois faire l'objet de mesures d'adaptation nécessitées par leur situation particulière (art. 73). Les differences de statut persisteront entre les DOM et les TOM. L'organisation institutionnelle de la Collectivité Territoriale de Mayotte se repprochait jusqu'à présent plus de celle d'un DOM que de celle d'un TOM.

3 Nous faisons abstraction icides"cas continentaux" comme Hongkong ou Macao, non comparables avec celui de la Guyane. 
pendance formelle, s'exprimant généralement par l'appartenance à l'ONU, est-elle une solution adéquate pour les mini-états? Ouvre-t-elle une meilleure voie pour attaquer leurs problèmes de développement (souvent plus aigus que ceux des pays du sud plus grands) que l'attachement continu à une métropole du nord? Nombre de cas de miniétats semblent le démentir, leur situation ayant empiré depuis l'obtention de la souveraineté nationale. C'est dans ce contexte que la position avancée par la France semble avoir une certaine légitimation: l'indépendance ne constitue pas la seule voie possible de décolonisation. Dans de situations déterminées, la solution contraire semble plus sensée, la départementalisation peut être "la solution française" de décolonisation. ${ }^{4}$ La position en faveur de l'intégration en général et de la départementalisation en particulier (Algérie) a été un trait caractéristique de toute l'histoire et de l'idéologie coloniales de la France et elle n' a pas été élaborée spécialement pour les petits pays. Pour l'empire en général, cette "solution française" s'est avérée intenable, trop à contre-courant de l'histoire, diamétralement opposée aux désirs d'émancipation des élites et des populations des pays dominés. C'est pourquoi Paris a finalement abandonné cette attitude rigide, pour entrer dans une relation plus flexible avec ses anciennes colonies, reconnaissant leur droit à la souveraineté formelle et préservant ainsi, de façon plus adaptée, ses propres intérêts. Mais pour les "confettis de l'empire" (J-C Guillebaud), c'est-à-dire les DOM et les TOM depuis 1962, la "solution française" a continué d'être défendue. Aujourd'hui, elle semble se poser ici dans des termes nouveaux. L'intégration des colonies (tentative en général vouée à l'échec par l'histoire) peut-elle prétendre être légitime dans des cas particuliers? Peut-elle constituer une voie pour résoudre les problèmes de développement et de "viabilitéu des petits pays?

\section{Une colonie "oubliée»?}

La décolonisation par incorporation était le postulat avancé par les gouvernements successifs de la France jusqu'en 1981, avec plus de force dans les années 70, lorsque le statut politique des TOM et surtout des DOM faisait d'eux des exceptions sur la scène internationale, au fur et à mesure que les petits pays du Tiers-Monde accédaient à l'indépendance. Paris admettait volontiers d'aller "à contre-courant du fameux sens de l'histoire " ${ }^{5}$ et considérait ce courant comme peu bénéfique pour les petits pays, ce qui paraissait souvent évident. Pour la Guyane Française en particulier, la départementalisation était défendue avec plus de résolution encore. N'était-ce pas une aberration pour cette colonie longtemps "oubliée", sous-peuplée, inexploitée, ne produisant rien, vivant totalement aux crochets de la métropole, de revendiquer un détachement de ses liens avec la "mèrepatrie", une émancipation en tant qu' Etat souverain?

4 François Luchaire: Droit d'outre-mer et de la coopération, Paris 1966, p. 115.

5 Secrétariat d'Etat aux DOM-TOM: La France et ses Départements d'Outre-Mer, Paris 1978, p. 32. 
En effet, l'héritage historique de 300 années de colonisation paraîssait confirmer la nécessité d'un traitement spécial de ce pays, le distinquant à première vue de toute autre situation et lui conférant (sur ce plan aussi) un caractère apparemment unique dans le monde. La Guyane: colonie "oubliée" mais toujours occupée, jamais abandonnée. Riche de potentialités mais jamais mise en valeur. Comment expliquer de telles contradictions? Sont-elles la clé pour comprendre non seulement l'histoire mais aussi le présent et les perspectives du pays? Justifient-elles une politique métropolitaine à son égard, qui ne peut être appréhendée avec les mesures communément employées pour aborder les difficultés du Tiers-Monde, les problèmes de développement et de sous-développement?

La première question ainsi soulevée pour une approche du cas de la Guyane est celle de la colonie longtemps "oubliée", ne sortant que depuis peu de temps "de la somnolence qui l'étreignait" (V. Giscard d'Estaing), ${ }^{6}$ étant de toute évidence un cas exceptionnel de pays colonisé. La Guyane était-elle une exception dans le colonialisme français? Elle y était inclue pratiquement dès ses débuts. ${ }^{7}$ L'historiographie officielle retient l'année 1604 pour son annexion par la France; il est peut-être plus réaliste de dater la prise de possession effective à la fin du 17 è siècle. ${ }^{8}$ En tout cas, le pays faisait depuis, déjà longtemps partie du premier empire colonial. Celui-ci était caractérisé par le contraste entre l'étendue de ses plus vastes possessions (surtout en Amérique du Nord), leur faible peuplement et leur peu de rendement économique pour la métropole. En revanche, les "îles", les nombreuses Antilles Françaises de cette époque jouaient un rôle considérable pour le mercantilisme parisien. La Guyane servait de plus en plus de point d'appui pour protéger le flanc sud du commerce lucratif avec la Caraïbe. Sa propre mise en valeur ne s'imposait guère, ce qui la distinguait nettement des colonies néerlandaises voisines (en particulier du Surinam), dont l'importance pour la Hollande était à peu près comparable à celle des Antilles pour le France. ${ }^{9}$ Ainsi s'expliquent les débuts du développement inégal des Guyanes. En 1763 cette situation changea radicalement. La France perdit presque tout son premier empire colonial. La Guyane devint la plus grande colonie restée attachée à la métropole et fut l'objet, dans la même année, de "l'émigration coloniale la plus importante qui ait eu lieu d'un seul coup sous l'Ancien Régime",${ }^{10}$ dont l'issue catastrophique marquait la première grande césure dans l'histoire du pays, lourde de conséquences pour son avenir.

Chronologiquement la deuxième empire colonial français commençait avec la prise d'Alger en 1830 et l'occupation et la "pacification" ultérieures de l'Algérie. En 1848 la

6 Dans sa préface au "Plan Vert “. Secrétariat d'Etat aux DOM-TOM: Le Plan Vert-Charte du Développement de la Guyane, Paris 1976, p. 1.

7 Pour le colonialisme français voir l'aperçu de Xavier Yacono: Histoire de la colonisation française, Paris 1973.

8 L'histoire de la Guyane est présentée en détail par Michel Devèze: Les Guyanes, Paris 1968 et par Arthur Henry: La Guyane Française. Son histoire 1604-1946, Cayenne 1974 (réédition).

9 Voir à ce sujet Cornelis Ch. Goslinga: A short history of the Netherlands Antilles and Surinam, La Haye 1979.

10 Michel Devèze: Les Guyanes, p. 62. 
possession de cette nouvelle colonie était consolidée. Elle avait déjà vu, dans les années précédentes, un afflux croissant de colons blancs qui s'appropriaient des terres et se mettaient à les exploiter. L'Algérie était en voie de devenir une colonie de peuplement et de mise en valeur, en fait elle allait être aussi la colonie la plus étendue du deuxième empire, réduisant nettement par là l'importance de la Guyane. Ce changement joua-t-il un rôle pour la deuxième cèsure cruciale dans l'histoire de la "France Equinoxiale"? Ce n'est peut-être pas un hasard, si la décision d'installer un bagne en Guyane intervint quelques années après l'achèvement de l'occupation territoriale de l'Algérie. Une nouvelle grande colonie pleine de potentialités, en voie de peuplement et d'exploitation était conquise. La plus grande colonie de ce qui était resté de l'ancien empire était désormais économiquement encore moins intéressante qu'auparavant. Avec l'extension ultérieure du deuxième empire, surtout à partir des années 1880, la Guyane devenait l'une parmi bien d'autres des colonies tropicales françaises, dont la plupart étaient en Afrique Occidentale et Equatoriale et où les obstacles à la mise en valeur étaient également considérables. De ce point de vue, la "colonie oubliée" en Amérique du Sud ne se distinguait guère de plusieurs autres "colonies oubliées" sur le continent noir, qui n'étaient que peu exploitées (si on considère les dizaines d'années de leur occupation) et qui n'eurent que peu d'importance économique pour la métropole jusqu'à l'entre-deux-guerres. Dans ce cadre général, la Guyane n'était pas un cas particulier du colonialisme français. Au temps du premier empire elle faisait partie des possessions plus grandes, ayant peu de signification économique. Au temps du deuxième empire elle faisait partie des possessions plus petites, ayant peu de signification économique.

Durant la première époque, l'intérêt de la France résidait surtout dans les "files". Durant la deuxiéme époque, son intérêt économique se limita assez longtemps à quelques grandes colonies, surtout l'Algérie, dans une moindre mesure et sur une base plus spéculative le Maroc, la Tunisie et l'Indochine. En somme, la colonisation française de la fin du 19è et des débuts du 20è siècle (phase de sa plus grande extension territoriale) était beaucoup plus "politique" qu'économique. ${ }^{11}$ Sur ce plan d'analyse, le cas guyanais n'a rien d'exceptionnel, il se situe très bien dans le tableau général. Mais c'est aussi ce tableau général qui fait ressortir les trois jalons essentiels, qui ont donné à la Guyane, vue maintenant à partir de son histoire plus concrète et non plus dans le contexte de l'histoire coloniale de la France, un destin assez singulier. Il s'agit des césures de 1763, 1848/52 et 1946.

La césure de 1763 a déjà été évoquée. C'était "le désastre de Kourou ", d'une tentative de colonisation massive précipitée sous l'Ancien Régime pour "compenser" la perte du premier empire colonial et pour établir un point fort de la France dans son territoire continental en Amérique du Sud. Cette tentative tourna en catastrophe avec des milliers de morts en quelques mois et conféra à la Guyane la réputation désormais chronique d'menfer vert", de "cimetière des Européens". ${ }^{12}$

11 Voir à ce sujet aussi Jean Bouvier: Les traits majeurs de l'impérialisme français avant 1914, dans: Le Mouvement Social (Paris), janvier-mars 1974, pp. 3-24.

12 L'entreprise (sur l'initiative du Ministre de la Marine, le Duc de Choiseul) avait été particulièrement mal préparée. "Avec la légèreté qui caractérise l'époque Louis $\mathrm{XV}$, on avait tout simplement oublié que toutes les co- 
Cette réputation joua son rôle lors des premières déportations sous la Révolution Française et dans les années suivantes. Elle se renforça davantage encore au moment de l'installation du bagne dans la colonie en 1851/52. La décision faisait suite aux conséquences de la libération des esclaves en 1848. Les Guyanes voisines et quelques Antilles assistèrent successivement à "l'importation" de travaillerus asiatiques (surtout de l'Inde) pour combler les besoins en main-d'oeuvre des économies de plantation. Pour la Guyane Française, Paris se décida à "importer" des prisonniers. Nous avons relevé plus haut le contexte général de l'extension du deuxième empire. Les intentions de "mettre en valeur" le pays par le travail pénal et de "réhabiliter" ainsi les bagnards (c'est la raison offciellement avancée) ont peut-être été sincères chez certains hommes politiques de bonne volonté. Dans la réalité, elles se révelèrent vite comme une hypocrisie cruelle. La France y voyait de plus en plus l'occasion de se débarasser de ses "déchets humains "13 (expression volontairement cynique), de vider ses prisons encombrées. La pratique du "doublage" est très parlante à cet égard. ${ }^{14}$ C'était le renoncement à toute fonction productive de la Guyane. Durant un siècle environ, le bagne fut la fonction principale (et aussi la plus longue) de la Guyane. C'était la perversion de la colonisation.

La troisième césure dans l'histoire de la Guyane est marquée par la départementalisation de 1946. Le pays était "exsangue "15 avant ce tournant, conséquence de son passé pénal auquel s'était encore ajouté le chaos officiellement toléré de "l'époque de l'or", qui fit de la Guyane pendant des décennies à la fois une prison, un cimetière et une "Californie" française. Mais après la deuxième guerre mondiale tout devait changer: "l'enfer vert" était incorporé à part entière à la métropole pour devenir "un département comme tous les autres". Soulignons que cette incorporation n'était pas imposée par Paris comme celle de l'Algérie cent ans plus tôt. Elle exprimait le désir des représentants des quatre vieilles colonies devenues des DOM et elle était revendiquée en premier lieu par les partis de gauche en Outre-Mer, pour mettre fin aux injustices coloniales et à la domination des "plantocraties" blanches locales. La Réunion, la Guadeloupe, la Martinique et la Guyane auraient bien pu devenir des TOM, comme la plupart des colonies dans l'union Fran-

lonisations fondées sur la main-d'oeuvre blanche en Guayane (anglaise, hollandaise, ou française) avaient jusqu'alors échoué. Si, encore, on avait envoyé en Guyane une émigration progressive, en commençant par quelques centaines d'hommes, qui auraient préparé les voies aux suivants en construisant des villages ... Mais on déversa d'un seul coup sur un pays qui comptait quelques centaines de Blancs, 10000 émigrants sans aucune expérience (1763-1764). Si encore on avait à l'avance préparé des stocks de ravitaillement . . Mais on envoya les malheureux colons (pour une moitié des Alsaciens et des Lorrains de langue allemande) avec des vivres de métropole, qui arrivèrent à Cayenne dans un état de conservation pitoyable. Les villages d'accueil n'étaient pas prêts: les émigrants durent souvent coucher par terre, sous un climat humide et dans un pays regorgeant d'insectes dangereux! En vain en transporta-t-on un certain nombre de la Terre Ferme dans des îlots plus salubres situés au large (les îles du Salut). Les maladies se déclenchèrent très vite, et une terrible épidémie ... mit le comble à la désolation. Sur le chiffre de 10446 arrivés, il y eut plus de 6000 victimes." Michel Devèze, Les Guyanes, p. 63-64.

13 Ian Hamel: Les Guyanais - Français en sursis?, Paris 1979, p. 42.

14 Cette pratique signifiait que les condamnés à moins de huit ans de travaux forcés devaient rester après leur libération en Guyane un laps de temps égal à la durée de leur peine. Les condamnés à plus de huit ans devaient rester dans le pays pour le restant de leur vie.

15 Gérard Brasseur: La Guyane Française. Un bilan de trente années, Paris 1978, p. 41. 
çaise. Leur intégration totale était une concession du gouvernement et de l'Assemblée Nationale à Paris, nullement contraints de la mettre en application. Mais une fois réalisé, que changeait le nouveau statut dans la vieille colonie? La Guyane est-elle devenue un département français comme les autres?

\section{Les effets de la départementalisation}

On sortirait du cadre de cet aperçu si l'on dressait un bilan détaillé des acquis et des défauts de la départementalisation depuis 35 ans. Le lecteur peut consulter les travaux de Cérard Brasseru, ${ }^{16}$ de Marie-José Jolivet ${ }^{17}$ et de Ian Hamel, ${ }^{18}$ qui constituent, chacun à sa manière, des utiles contributions sur ce point. Retenons seulement que même pour les adversaires les plus déterminés du statut politique actuel de la Guyane, il est impossible de nier les progrès considérables apportés par l'incorporation à la métropole, surtout dans les domaines de la santé, de l'éducation et du niveau de vie général. La Guyane se place ici probablement en tête de tous les pays sud-américains. ${ }^{19}$ D'un autre côte, il ne faut pas oublier qu'il s'agissait seulement d'une très petite population, n'atteignant aujourd'hui que 75000 habitants environ. ${ }^{20}$ Le coût, pour la France, pour "entretenir" la Guyane n'était et n'est pas très élevé; le DOM ne représentait que 0,1 à $0,2 \%$ des dépenses dans le budget français dans la deuxième moitié des années 70 . De plus, $90 \%$ à peu près de l'argent public transféré en Guyane reviennent en métropole sous la forme de revenu privé. Cela est du au caractère "tertiaire" de l'économie guyanaise, celle-ci étant essentiellement composée "tertiaire" de l'économie guyanaise, celle-ci étant essentiellement composée de l'administration et du commerce d'importation, l'un étant le pendant de l'autre. ${ }^{21}$ Cette structure rend difficile, par son principe même, toute tentative de déve-

16 Id.

17 Marie-José Jolivet: La question créole. Essai de sociologie sur la Guyane Française, Paris (ORSTOM) 1982; ici cité dans sa première version: Essai de sociologie sur la Guyane Française, Paris (ORSTOM) 1978 (Thèse de Doctorat non-publiée).

18 Ian Hamel, op. cit.

19 En moyenne, son niveau de vie est proche du niveau de vie français ce qui s'exprime par le nombre de voitures, de postes de radio et de télévision, de téléphones et d'autres articles de consommation élévée par habitant. En 1979 il y avait en Guyane plus de voitures par habitant qu'en France. Voir à ce sujet aussi André Calmont: Cayenne - La ville et sa région, Bordeaux-Talence 1978.

20 Le nombre d'habitants s'accroît approximativement de 2000 personnes par an au début des années 80 .

21 En 1974, 60,4 \% de la population active ętaient occupés dans le secteur tertiaire (commerce, transports, banques, services), $21,5 \%$ dans le secteur secondaire (industries de transformation, bâtiments et travaux publics, électricité, gaz et eau) et $18,1 \%$ dans le secteur primaire (agriculture, sylviculture, chasse et pêche, industries extractives). La Guyane comptait en 1976 environ 10 \% de chômeurs selon les chiff res officiels, mais ce pourcentage ne réflétait qu'une partie de la réalité. ". . a à côté des emplois répertoriés fleurissent de nombreuses activités à temps partiel qualifiées de 'jobs' qui, sans nourrir pleinement leur homme, dépannent les chômeurs et assurent des revenus appréciables aux autres - et évidemment non déclarés à la Sécurité sociale et aux impôts." Gérard Brasseur: La Guyane Française, p. 141. En 1980, le député gaulliste du département, H. Riviérez, situait le taux de chômage à près de $30 \%$. Le Monde, 31. 12. 1980. 
lopper des activités productives. Elle est l'héritage du passé "pénal" du pays, mais elle est aussi conséquence de la départementalisation schématique, faisant de la Guyane "un département comme les autres" et tenant peu compte de sa spécificité socio-économique. De cette façon, le niveau de vie guyanais repose sur des bases artificielles. Il n'est pas le résultat de son propre développement mais presque entièrement dépendant du "cordon ombilical" qui lie le DOM à la France. Nous ne retenons pas l'hypothèse qu'il s'agissait dès le début d'un sous-développement voulu par Paris pour mieux garder le pays dans le giron français, parce que le départementilisation, désirée par la Guyane, se présentait en 1946 comme la meilleure alternative et ne fut contestée par personne pendant assez longtemps. Néanmoins, de l'histoire ancienne et récente de la Guyane est issue une société locale largement "parasitaire", "assistée", vivant aux crochets de la métropole. La responsabilité en incombe à la France. Sur le plan guyanais, cette situation rend problématique tout essai pour s'affranchir de la tutelle métropolitaine. Non seulement les Métropolitains "chasseurs de primes" dans le département et une petite élite créole locale, mais aussi beaucoup de Guyanais auraient à perdre au moment d'une "rupture" avec Paris. L'arrêt ou la diminution des transferts financiers français pourraient se faire sentir de manière plus douloureuse dans le DOM. Une alternative à ce dilemme serait le développement d'une économie plus productive laquelle semble en effet s'amorcer depuis quelques années, mais qui reste tout de même encore à l'état embryonnaire. La gauche et les nationalistes en Guyane seront-ils prêts à envisager à terme une fin de la "Guyane des bureaux", une réduction radicale du tertiaire, une "prolétarisation" des Guyanais pour une période transitoire, afin de pouvoir revendiquer leur propre destin, indépendant de la France, avec plus d'autorité? Bien sûr la question du développement d'une économie productive en Guyane ne touche pas seulement les intérêts guyanais. Elle peut aussi être posée au gouvernement parisien. La France peut-elle se permettre un "jardin botanique" en Amérique du Sud?22 A quoi lui sert la Guyane? Quel est son intérêt dans son DOM le plus étendu?

\section{L'intérêt de la France en Guyane}

Cette question est devenue d'une certaine actualité depuis 1975, lors du lancement du "Plan Vert" à Paris, qui eut un écho remarquable dans la presse française et internationale, auquel les propos et les actions du premier ministre et du secrétaire d'était aux DOM-TOM de l'époque, Jacques Chirac et Olivier Stirn, n'étaient pas tout à fait étrangers. Malgré le contraste entre les "grands projets" et les "petites réalisations " ${ }^{23}$ et malgré les commentaires de plus en plus sceptiques publiées dans les années suivantes, allant

22 C'était la qualification employée par un haut fonctionnaire de la Direction Départementale de l'Agriculture à Cayenne, lors d'une conversation avec l'auteur en septembre 1979.

23 Jean Octobre: Le plan de développement de la Guyane: Grands projets, petites réalisations, in Le Monde, 3. 8. 1977. 
jusqu'au constat "d'échec" du plan, ${ }^{24}$ la Guyane n'a plus depuis disparu de la scène, elle ne s'est plus "endormie", elle n'est plus un pays "oublié", et ce changement semble être définitif.

Dans un travail plus large ${ }^{25}$ nous avons analysé de façon détaillée, champ par champ, les intérêts actuels et potentiels de la France en Guyane, partant des domaines principaux évoqués dans le "Plan Vert". Cette analyse ne peut pas être reproduite ici. Le lecteur devra consulter les ouvrages cités dans les notes et parmi eux surtout l'atlas de la Guyane, réalisé conjointement par le CEGET à Bordeaux-Talence et l'ORSTOM à Paris. ${ }^{26}$ Relevons simplement que les ressources forestières sont de loin la matière première principale du pays, dont l'exploitation demeure un intérêt constant pour la France. Bien que, l'installation d'une industrie de pâte à papier ait été annoncée de manière un peu trop précipitée par Jacques Chirac et Olivier Stirn au milieu des années 70 et qu'il se heurtât aux prix oscillants du marché mondial et aux différences entre Paris et les entreprises américaines intéressées quant au financement de l'infrastructure à mettre en place dans le DOM, on ne peut pas exclure un regain d'actualité de ce dossier dans l'avenir. (Le dernier gouvernement giscardien l'avait annoncé pour la fin des années 80). Hormis cela, l'exploitation "conventionnelle" du bois guyanais augmente à un rythme accéléré depuis quelque temps. Il est à espérer que celle-ci se maintiendra dans des limites raisonnables. Une répétition de la manière avec laquelle la forêt est exploitée - et détruite - actuellement dans des autres régions équatoriales entraînerait très vite les mêmes dévastations irréversibles en Guyane, comme elles se sont produites déjà sur des superficies beaucoup plus grandes au détriment de l'écologie mondiale.

Quant aux ressources minières, à la différence de ce qu'on pouvait attendre après la lecture du "Plan Vert", la Guyane n'en semble pas dotée outre-mesure. Au moins à en juger pour les informations jusqu'à présent rendues publiques, les réserves de bauxite demeurent limitées et les autres indices pas trop prometteurs. Le kaolin en constitue peut-être une exception.

L'exploitation des ressources maritimes est caractérisée surtout par la pêche à la crevette. Essentiellement aux mains d'armements étrangers (américains et japonais pour la plupart), elle est en train de voir les débuts d'une plus grande participation française. Mais souligons ici que cette matière première ne constitue pas un intérêt primordial pour la métropole, elle n'est pas une ressource dont la France est déficitaire, elle n'alourdit donc pas sa facture d'importation. La pêche industrielle représente plutôt un facteur d'intérêt pour la Guyane et pour son propre développement. En revanche, une autre "ressource maritime", la zone de 200 milles au large de la côte guyanaise intéresse la France également, bien que son exploitation ne soit pas achevée et sa valeur économique reste encore à prouver.

24 Jean-Emile Vié: L'échec du Plan Vert en Guyane, in Le Monde, 1. 7. 1977.

25 Frank Schwarzbeck: Französisch-Guayana, pp. 153-218.

26 CEGET/ORSTOM (Centre d'Etudes de Géographie Tropicale/Office de la Recherche Scientifique et Technique Outre-Mer): Atlas des Départements Français d'Outre-Mer, 4. La Guyane, Paris/Bordeaux-Talence 1979. 
Le centre spatial de Kourou est trop connu pour être présenté ici en détail. Outre les articles dans les revues scientifiques le lecteur consultera utilement l'ouvrage de P.-M. Découdras et les passages correspondants dans la thèse de M.-J. Jolivet. Dans notre travail $^{27}$ nous avons adhéré à l'analyse de Marie-José Jolivet sur l'impact psychologique peu bénéfique de la nouvelle ville de Kourou dans le cadre guyanais. ${ }^{28}$ Dans sa forme actuelle elle fait figure comme corps étranger, de "ville blanche" dans un pays créole. De surcroît, elle est structurée d'une façon telle que les différences sociales et raciales y trouvent une projection géographique avec des traits presque caricaturaux. Quant à l'importance économique du CSG, elle reste relativement limitée. Malgré toutes les déclarations lors de son implantation, und base spatiale ne pouvait pas faire "décoller" la Guyane, remplacer par elle même une économie productive manquante. Ce jugement n'est pas contredit par le fait que le centre spatial et le "nouveau Kourou" ont sans doute apporté au pays des améliorations en matière d'infrastructure. ${ }^{29}$ Mais cette constation serait incomplète sans mentionner également le brusque arrêt de la bonne qualité de la "route nationale" après le champ de tir de la fusée européenne "Ariane". Immédiatement après, la route se rétrécit, les trous se creusent. Autre facteur caricatural autour du CSG . . . Evoquons finalement en quelques mots la question de l'importance de la base spatiale hors de la Guyane. Sa signification économique et technologique pour la France et pour l'Europe Occidentale est indubitable. At-elle aussi une importance militaire?

Des propos de l'ex-président Giscard d'Estaing en février 1979 donnaient l'impression que son gouvernement ne voulait pas s'obliger à exclure une fonction potentielle du DOM sud-américain pour la "force de frappe", en dépit de son adhésion au "Traité de Tlatelolco", visant l'interdiction des armes nucléaires en Amérique Latine. ${ }^{30}$ Mais l'examen minutieux de ce traité fait ressortir clairement qu'un usage de ces armes dans sa zone d'application est effectivement incompatible avec ses prévisions. Cela mis à part, on voit mal l'intérêt nucléaire et stratégique de la France en Guyane. Sur quelle cible éventuelle voudrait-elle tirer depuis Kourou, vu le fait que la force de dissuasion ne dispose que de fusées à moyenne portée? Une autre question concernait le transport des matières nucléaires de la France via les DOM caraîbéens dans le Pacifique Sud, empruntant ainsi le chemin le plus court pour atteindre le centre d'expérimentation en Polynésie. Ce trans-

27 Frank Schwarzbeck, op. cit., pp. 197-201.

28 Marie-José Jolivet: Essai de sociologie, pp. 532-536.

29 Cf. Pierre-Marie Découdras: Kourou - Ville spatiale, Bordeaux 1975 (Thèse de Doctorat non-publiée).

30 Giscard répondait à la question d'un journaliste de "La Lettre de la Nation" lors de sa conférence de presse du 15 février 1979. Interrogé si sa signature imminente du protocole 1 du Traité de Tlatelolco voulait dire que "le département français de la Guyane est absolument exclu de la zone nucléaire française", le Président disait que "le droits de souverainété, notamment de souveraineté militaire française, pour ce qui concerne la Guyane, ne seront pas modifiés par ce protocole." Le Monde, 16. 2. 1979. Le Traité de Tlatelolco (du nom du quartier de Mexico où se trouve le ministère mexicain des affaires étrangères) a été approuvé par 21 états latinoaméricains en 1967. Dans son annexe, il contient deux protocoles qui s'adressent aux puissances non-latinoaméricaines: les signataires du protocole 2 sont les puissances nucléaires qui s'engagent à ne pas employer les armes nucléaires contre les signataires du pacte. Les signataires du protocole 1 sont les pays non-latino-américains qui administrent des territoires dans la zone du traité et qui s'engagent à respecter ses prévisions pour ces territoires. La France a signé le protocole 2 en 1973 et le protocole 1 en 1979. 
port s'effectue par les Antilles ainsi que par l'Amérique du Nord; il est compatible avec le traité de Tlatelolco. Finalement il reste pour la Guyane la fonction militaire: servir de base de lancement des satellites d'observation. Mais tout compte fait, pour l'instant du moins, la signification économique du CSG paraît l'emporter largement sur son importance stratégique.

De l'analyse précédente semble ressortir la prépondérance actuelle du CSG comme facteur d'intérêt pour la métropole suivi par l'exploitation forestière. Mais pour arriver à une vision plus précise, il convient d'élargir le faisceau d'interrogations et d'y inclure les autres DOM et TOM. Y-a-t-il des fonctions complémentaires des différentes possessions d'outre-mer, qu'on ne peut saisir que dans leur contexte d'ensemble et non par l'étude de la problématique d'un seul département ou territoire?

Cette question a été soulevée en 1976 par un article de l'économiste français Jean-François Troussier, publié dans la Pevue Tiers-Monde. ${ }^{31} \mathrm{Y}$ figure en premier lieu l'interrogation sur le rôle potentiel de débouché. La Guyane est-elle intéressante comme marché pour l'écoulement des produits métropolitains? La faible population du pays infirme aussitôt l'hypothèse, et il en va de même (en dépit des dimensions plus élargies) pour tous les DOM-TOM. Les petites entreprises françaises pour lesquelles la Guyane et les DOM-TOM pourraient jouer un rôle dans leurs chiffres d'affaires, ne peuvent avoir une capacité de pression suffisante pour influencer la politique métopolitaine Outre-Mer. Pour les grandes entreprises, qui en pourraient avoir davantage, la Guyane et les DOMTOM ne sont pas suffisamment importants.

Même observation de principe pour la question du placement des capitaux dans les "confettis de l'empire". Dans le Tiers-Monde, les capitaux français se concentrent surtout dans les anciennes colonies d'Afrique noire. A cause du coût élevé de la main-d'oeuvre locale, les gouvernments parisiens ont jusqu'à présent plutôt connu des difficultés pour inciter des hommes d'affaires à investir dans les DOM-TOM. Des changements sur ce point ne se produisent que lentement.

L'empire éparpillé ne jouait pas non plus un rôle considérable comme fournisseur de matières premiéres dans le passé, le nickel de la Nouvelle-Calédonie faisant figure d'exception. Mais cette situation commence à changer depuis quelque temps. Les gouvernements français se sont de plus en plus rendus compte des potentialités éventuelles des départements et territoires d'outre-mer, surtout depuis le début de la crise de l'énergie (au moins pour atténuer son impact), des études sont entreprises presque partout, tant au Pacifique Sud et en Océan Indien, que dans les Caraîbes et au large de la côte sud-américaine. $^{32}$

C'est sur une quatrième interrogation, celle d'une fonction possible des DOM-TOM comme réservoir de main-d'oeuvre, que J.-F. Troussier fait entrer l'hypothèse de leur complémentarité. ${ }^{33}$ Peut-il être intéressant pour la France de ne pas les industraliser afin

31 Jean-François Troussier: La Guyane en questions, in Revue Tiers-Monde (Paris), 1976, pp. 721-737.

32 Cf. Commissariat Général du Plan: Rapport du Comité DOM-TOM, préparation du 8e Plan, Paris 1980.

33 Jean-François Troussier: La Guyane, p. 737. 
d'utiliser en métropole leurs travaillerus émigrés? Et peut-il étre lucratif de réaliser en même temps des "grands" projets d'investissement "dans un pays vide, aux grandes potentialités, comme la Guyane" ?? ${ }^{34}$ L'hypothèse paraît séduisante à première vue. Elle a été formulée peu de temps après le lancement du "Plan Vert", mais elle e été démentie entretemps par les faits. La Guyane n'a pas vu jusqu'à présent les "grands" projets trop vite annoncés, la crise de l'économie française dans les années 70 n'a pas rendu urgente une exploitation à grande échelle des matières premières du département comme Troussier l'avait supposé en 1976. En ce qui concerne les "Domiens" en France, leur afflux s'est accru en effet de plus en plus depuis le début des années 60. En 1980 on dénombrait 400000 immigrés environ, venus par l'intermédiaire du BUMIDOM, et plus encore par l'immigration spontanée et non-organisée. ${ }^{35}$ Mais si cette immigration était en grande partie engendrée par le chômage augmentant outre-mer, elle n'était guère une nécessité pour le marché du travail en métropole. Au temps de l'essor économique dans les années 60 la France connaissait egalement l'immigration croissante des étrangers, qui sont aujourd'hui plus de quatre millions. Et avec la récession à partir de 1973, la France était confrontée à un chômage toujours plus problématique et à la présence de nombreux immigrants du Tiers-Monde en plus, avec toutes les difficultés qui en découlent.

Aux quatre hypothèses précédentes (confirmées ou invalidées à différents degrés) il faut en ajouter une cinquiéme pour comprendre l'intérêt de la France dans ses DOM-TOM. Ont-ils des fonctions pour sa politique extérieure et sa défense?

Le rôle du CSG en Guyane a déjà été évoqué. Un atoll en Polynésie sert de champ d'expérimentation pour les essais nucléaires de la "force de frappe". La Réunion (beaucoup plus que Mayotte et les petites îles dans le canal de Mozambique) revêt une position stratégique importante pour surveiller la "route du pétrole", empruntée par les tankers venant du golfe persique et contournant le cap de la Bonne Espérance. La France a-t-elle pu éviter de rétrograder "en-deça du rang de première puissance de second ordre" grâce à ses "confettis de l'empire", comme l'a affirmé Jean-Emile Vié? ${ }^{36}$ Ses possessions dispersées "aux quatre coins du monde" justifient-elles son "intervention dans la solution des grands problèmes" "?7 Peut-être la formule est un peu exagérée. En tout cas, cette "présence française" n'a jusqu'à présent, guère causé des ennuis à Paris sur la scéne internationale, quelques protestations éposidiques de la "Communauté Caraïbe" et de l'Organisation de l'Unité Africaine mises à l'écart. De ce côte, la continuation de l'apartenance des DOM-TOM à la métropole ne semble pas encore être sérieusement mise en cause. A l'inverse, elle ne fait plus l'unanimité dans les populations des pays concernés. Des minorités de plus en plus larges revendiquent un destin propre, un destin "national" pour la Martinique, la Guadeloupe, la Réunion et la Guyane. Revenons ainsi à son cas. La Guyane peut-elle envisager une telle voie, pourvu que la France y consente? Peut-elle

34 Ibid.

35 Commissariat Général du Plan: Rapport du Comité DOM-TOM, p. 81.

36 Jean-Emile Vié: Faut-il abandonner les D.O.M.?, Paris 1978, p. 134.

37 Ibid. 
devenir un Etat caraïbéen/sud-américain? Y-a-t-il cette alternative à la dépendance permanente de la "mère-patrie«?

\section{Quelle alternative pour la Guyane?}

Toute discussion sur cette question paraît sans objet si l'on en croît l'avertissẹment de Jean-Emile Vié, ancien secrétaire général pour l'administration des DOM, rue Oudinot, formulé dans son livre paru en 1978: en cas de détachement de la Guyane de la métropole, "son annexion par le Brésil risquerait de n'être qu'une formalite". ${ }^{38}$ Cette assertion est assez répandue (et peut-étre pas tout à fait innocente) parmi les Français métropolitains dans le DOM; on peut même y entendre parler des cartes de la Guyane au Brésil, sur lesquelles des parties sont hachurées, et démarquent ainsi les prétentions territoriales du "voisin géant". Ces cartes n'existent pas, plus qu'il n'y a de menace annexioniste brésilienne, ni de "risque brésilien" de ce genre. Brasilia aurait peu à gagner mais beaucoup à perdre sur le plan international en essayant "d'empocher" ainsi la Guyane. Mais même si cette possibilité semble éloignée de la réalité et plus proche de cauchemars volontairement entretenus, on ne peut pas écarter pour autant un autre "risque brésilien" du DOM, vu le dynamisme d'expansion et de "conquête" de l'Amazonie déployé par le Brésil.

La Guyane Française et les Guyanes voisines risqueraient d'être satellisées économiquement, subissant le sort qu'ont connu l'Uruguay et la Bolivie, des Etats frontaliers pourtant bien plus grands. Pour l'instant, ce risque n'est pas encore imminent, le Brésil étant confronté actuellement à d'énormes difficultés qui ralentissent son rythme d'exploitation et d'avancement dans le bassin amazonien. Mais cela ne durera pas toujours. La "frontière mobile" (expression courante au ministère des affaires étrangères à Brasilia) s'approchera des Guyanes. ${ }^{39}$

Pour se soustraire au danger d'être satellisée à terme (sand aucune violation de ses frontiéres) par son voisin du sud, la Guyane aurait intérêt à explorer les prémisses d'une intégration dans la Caraïbe, d'une coopération plus étroite avec les autres petits pays de la région, avec lesquels elle a beaucoup de points en commun et qui sont en quelque sorte ses "pays frères". Cette évidence n'est pas partagée par tout le monde, même pas par tous les indépendantistes et autonomistes dans le DOM. Cela s'explique par l'isolement historique de la colonie, qui se poursuit jusqu'aujourd'hui et qui est conditionné par la politique française. Cet isolement a, sur le plan politico-psychologique, des conséquences pas toujours faciles à surmonter.

38 Ibid., p. 137.

39 Pour une analyse of ficielle du programme de développement dans le Territoire Fédéral brésilien de l'Amapá, voisin de la Guyane, au milieu des années 70 cf. Ministério do Interior - Território Federal do Amapá: II Plano Nacional de Desenvolvimento. Programa de Acão do Governo para o Território do Amapá (1975-79), Brasilia 1975. 
Cela s'explique aussi par la faible population de la Guyane et par le traumatisme obsédant de perdre son identité par trop d'échanges avec l'environnement, cette "peur des contacts" s'appliquant même à la Martinique et la Guadeloupe. En fait, l'ignorance de beaucoup de Guyanais de leurs pays voisins est frappante. D'une façon à peine exagérée on pourrait dire que presque tous connaissent Paris, mais presque personne n'a été à Georgetown ou à Port-of-Spain. Cet "attachement à la métropole", valable aussi pour l'Unité Guyanaise et le PSG, est incompatible avec la revendication de l'indépendance et de l'autonomie. La Guyane est un petit pays créole ${ }^{40}$ comme le sont aussi la plupart des excolonies britanniques dans la région qui forment la "Communauté Caraïbe". Les Guyanais devraient connaître leurs problèmes de développement et la situation de leur marché commun, le CARICOM, parce que c'est cette alternative à laquelle ils pourraient être confrontés, une fois acquise l'indépendance formelle en tant qu'Etat souverain. Il n'est pas possible d'aborder ici de façon approfondie la problématique de "grandeur/petitesse et développement/sous-développement" et de là les difficultés du CARICOM, comme nous l'avons fait dans un cadre plus large. ${ }^{41}$ Cette analyse fait clairement ressortir que la coopération regionale n'a apporté aux pays membres jusqu'à present que des améliorations plutôt modestes. Elle favorisait surtout les "quatre grande" (Guyana, Trinidad/Tobago, Barbade, Jamaïque) du CARICOM, groupe auquel la Guyane appartiendrait, une fois entrée dans cette alliance, en raison de son étendue territoriale et de ses ressources. Mais les difficultés pour un développement auto-centré des pays caraïbéens ne sont pas que de nature économique. Leur choix d'une stratégie propre se heurte aussi à ces obstacles politiques internationaux, en particulier à la tendance actuelle des EtatsUnis à percevoir dans toute tentative de s'affranchir des structures socio-économiques léguées du passé colonial "un deuxiéme Cuba", qu'ils s'efforcent par conséquence de saboter (comme à Grenade, au Nicaragua, ou au Salvador).

C'est pourquoi le "Communauté Caraïbe" reste indispensable pour les "mini-états" de la région, malgré tous ses défauts économiques. Elle donne aux petits pays une certaine protection politique en tant qu'entité plus grande, augmentant un peu leur poids international face aux états voisins. Même dans l'hypothèse d'une atténuation future de l'antagonisme Est-Ouest dans l'hémisphère, le CARICOM restera nécessaire. Et une Guyane indépendante ne pourrait pas se passer de coopérer avec ses membres, sous une forme ou sous une autre.

Quel avenir pour la Guyane? Pour l'instant le pays ne peut pas encore se passer ni du

40 Ce terme désigne les 4/5e de la population guyanaise. On entend par "Créoles" les descendants des Noirs introduits aux Caraibes par la traite aux XVIIe et XVIIIe siècles. "Des Blancs ont pu se meler à eux, si bien qu'anthropologiquement toutes les teintes des peaux se rencontrent, mais dans l'ensemble les caractères négroides l'emportent." Gérard Brasseur: La Guyane Française, p. 32.

41 Frank Scharzbeck: Französisch-Guayana, pp. 263-293. Des contributions importantes sur ces questions complexes ont été fournies par des chercheurs caraibéens. Voir (entre autres) Vaughan A. Lewis (éd.): Size, SelfDetermination and International Relations - The Caribbean, Kingston 1976; Basil A. Ince (éd.): Contemporary International Relations of the Caribbean, St. Augustine 1979; Lloyd Best: Size and Survival, in N. Girvan/O. Jefferson (éds): Readings in the Political Economy of the Caribbean, Kingston 1974, pp. 29-34. 
soutien économique ni de la protection internationale de la France. Paris doit aider le mieux possible ses DOM-TOM à préparer leur propre destin. La métropole le doit en particulier á la Guyane, à son ancienne "colonie oubliée", à "cendrillon ", à "l'enfer vert" et au "cimetière des Européens", au pays qui dans tous ces rôles, a longtemps fait les frais des caprices de la colonisation.

Paris ne devrait pas imposer l'indépendance et "lâcher" la Guyane aussi longtemps qu'une majorité de sa population n'y consentira pas. Mais il devrait tout faire pour éviter une future décolonisation bâclée ici comme dans les autres "confettis de l'empire" restants, ainsi qu'elle s'est produite ailleurs.

En 1960 le Général de Gaulle déclarait à Cayenne qu'il "est dans la nature des choses qu'un pays comme la Guyane, qui est en quelque sorte éloigné et qui a ses caractéristiques propres, jouisse d'une certaine autonomie proportionnée aux conditions dans lesquelles il doit vivre". Avant les élections en 1981 le programme présidentiel du candidat François Mitterrand promettait "la gestion des affaires locales dans la perspective d'un avenir librement débattu“ aux populations des DOM. (Le Monde, 8. 5. 1981) Après les élections, Gaston Defferre, ministre de l'intérieur et de la décentralisation (et ministre de la France d'Outre-Mer 25 ans plus tôt, lors de la "loi cadre") déclarait: "Les départements d'outre-mer sont encore soumis à une exploitation coloniale; la population autochtone y est misérable, elle a besoin du concours de la France. Si on leur proposait un statut particulier, ils pourraient croire que la France veut les abandonner ou commence à les abandonner, ce qui n'est pas le cas." (Le Monde, 17. 7. 1981)

D'après cette déclaration, pour le moment du moins le gouvernement ne semble pas disposé à concevoir un statut spécial pour les DOM, à la différence de ce qu'il fait pour la Corse. Mais même en incluant les départements d'outre-mer dans la grande réforme des collectivités locales de 1982, il devrait tenir compte de leurs spécificités. Les changements institutionnels prévus pour 1983 constituent un pas dans cette direction. Ici il ne s'agit pas d'un problème "régional" de la France. Les Réunionnais, les Guadeloupéens, les Martiniquais et les Guyanais ne sont ni des Bretons ni des Corses. Ils ne sont pas des Européens, ils vivent dans un environnement différent. Comme les autres trois vieilles colonies la Guyane est un pays du tiers-monde. Bien sûr francophone, imprégné de la culture et de l'histoire françaises, à l'avenir certainement aussi un pays ami de la France. Mais c'est un pays appelé à décider de son propre destin et pas un département français comme les autres. 


\section{The Privatization of State Pension Schemes in Chili}

\section{By Maximilian Fuchs}

The history of social security run by the state in Chile goes back to the 1920s. Ever since, a sophisticated system of social security provisions with a high rate of coverage has been developed.

As is well-known, the political and economic framework changed dramatically after the military coup in 1973 . The new political leadership began to do away with the traditional structures and institutions in politics and the economy. The dominant model and guidelines, which the new military junta followed, have been essentially formulated by members of the so-called "Chicago school", the doyen of which is the Nobel-Prize winner Milton Friedman.

In a nutshell, the leading principle was to create the conditions for the functioning of a free market economy, where state activities are reduced to a necessary minimum. Needless to say, these goals could not be attained without fundamental changes in the field of social security. Neo-classical criticism of the welfare state prompted high-ranking officials in the Chilean public administration to reorganize the social security system by abandoning the classical pattern of social security.

The most striking measure following the new directions was the new act on old age and invalidity insurance (Decreto Ley Nr. 3500, of 13. 11. 1980). The new legislation replaces the old public scheme by introducing a private insurance model administered by private companies. Employees and (on a voluntary basis) self-employed persons are free to choose to which insurance company contributions should be made. In this way one hopes to stimulate competition on the insurance market. As a principle benefits in the case of old age and invalidity (including benefits for survivors) are dependent on the contributions paid by the insured and the interest on these contributions.

The article analyses the new legislation and attempts to show its consequences and impact on the social development of the country.

\section{Guiana - A French Department like the Others?}

\section{By Frank Schwarzbeck}

French Guiana is today the only continental country in the Third World which has a considerable surface area and is still completely part of a European State. It is the largest of the remaining French overseas departments and overseas territories, which were formally integrated into metropolitan France after the Second World War. This paper presents Guiana's unusual history, the consequences of departmentalization, France's interests in this country, and its future prospects. 
Guiana is sometimes characterized as an exceptional case, because it is one of the oldest French colonies, having nevertheless at no time really been economically utilised by the metropolis. The history of French colonial empires, in the 17th/18th and in the 19th/ 20 th centuries, however, reveals that there have been numerous possessions with little economic value for France for a long time, Guiana being no exception but well within this general picture.

In contrast to that, it has been the object of three decisions of the Paris government, which have particularly marked its history. In 1763, Guiana was chosen for a colonial mass-settlement. This attempt turned into a catastrophe and brought to the country its reputation as a "Green Hell". In 1852, it became a penal colony, being for almost 100 years France's outlet for its "human garbage" - the "Green Hell" in South America served to empty the over-congested French prisons. In 1946, finally, the sapped and "anaemic" colony became by law a part of France. From then on it was officially presented as "a French department like the others".

As a consequence of departmentalization Guiana probably has the highest living standard in South America. Nevertheless, this proud claim by the French government must be contrasted with the fact, that the overseas department has only a very small population (at present, about 75,000) and is no substantial burden on the State budget of France. The standard of living in Guiana is almost entirely dependent on external financing. After departmentalization the tertiary sector of the local economy has become even more dominant, being essentially composed of administration and commercial importation. The state of its still largely unproductive economy is a serious handicap for any attempt of Guiana to achieve independence as a Caribbean/South-American State.

The most important French interest in this country for the moment is the Space Centre at Kourou, where the European rocket "Ariane" is launched. Among the natural resources of the department timber stands out - $90 \%$ of Guiana's surface being covered by tropical rain forest. Minerals and maritime resources are less important. The cost for exploitation is relatively high due to the lack of infrastructure. For the time being, Guiana seems to be more a reserve than a necessary overseas potential for France.

The final part of this article discusses possible alternatives to the present political status. A former high official of the French Overseas State Secretariat has put forward an often quoted warning: If the department should break ties with France, "its annexation by Brazil risks being a formality only". Of course this is more an intimidation, there is no real "Brazilian risk" of this kind. But Guiana could well one day be an object not of annexation but of gradual economic "absorption" by its giant neighbour, provided that the Brazilian "conquest" of Amazonia continues, a conquest the consequences of which are already felt in much bigger states bordering the Amazon basin, as Paraguay and Bolivia.

In order to avoid being eventually drawn into Brazil's orbit, Guiana should turn more towards the Caribbean. The attempts of integration of the small countries in this region, however, do not allow much optimism. After ten years the "Caribbean Community" and its "Common Market" (CARICOM) have brought only modest progress to their mem- 
bers, the differences between the richer and the poorer CARICOM-countries even increasing. Economic shortcomings as well as political difficulties were responsible for CARICOM's relative failure, the political difficulties being mostly engendered by the effects of the East-West-conflict in this part of the Third World.

This is why Guiana cannot dispense with its ties to Paris for the forseeable future. But the country will only benefit from them if the French government does accept the fact that by helping to build a more productive local economy and by transferring more political competences to the Guianese élite, it must prepare self-determination for the last continental overseas possession in Latin America, which cannot forever retain its present political status.

\section{The Development of the Jamaican Workers' Savings and Loan Bank in the Seventies}

\section{By Dieter Semmelroth}

In Jamaica there was growing criticism of the performance of foreign banks, which monopolized commercial banking in the early seventies. The reform - minded Prime Minister Michael Manley promoted the plan to establish a Jamaican - owned and run financial institution. In 1973 the Workers' Savings and Loan Bank (WSLB) was founded. The shares of this bank are held by major trade unions, co-operatives, the government and the public. The WSLB was inaugurated to function as a fully commercial banking institution with the aim of serving all segments of Jamaican society. Moreover it was expected that the WSLB should finance and develop programs and projects which promise high social and developmental return.

However, the overall performance of the WSLB during the seventies was rather disappointing. After having overcome some serious problems in its early years, the WSLB has managed in the meantime to achieve a middle position in the Jamaican banking business. But an evaluation of WLSB's savings and lending policy reveals that it does not differ significantly from that of its multinational competitors. The bank has largely failed to develop new and innovative projects and programs. Trade unions and co-operatives are increasingly dissatisfied by the "conservative" policy pursued by the WSLB management. Like other workers' banks in Latin America and the Caribbean, the Jamaican WSLB has not been able to meet the high expectations connected with its establishment. 